\title{
Refractory Persistent Pulmonary Hypertension of the Newborn Responsive Only to Neuromuscular Blockade
}

\author{
Cristina Delco ${ }^{\mathrm{a}, \mathrm{b}}$, Oliver Karam ${ }^{\mathrm{a}}$
}

\begin{abstract}
We discuss the case of a premature newborn boy presenting with severe persistent pulmonary hypertension (PPHN) at 48 hours of life in the context of Staphylococcus aureus sepsis. PPHN was refractory to classical treatment (oxygen, control of acid basic balance, sedation, high frequency oscillation ventilation, inhaled nitric oxide and antibiotics). We observed an improvement only after administration of a curare. Despite much progress in the management of this entity, a clear and well defined therapy is still missing and especially when PPHN is refractory to classical treatment. The use of neuromuscular blockers in the treatment of this kind of PPHN has been described and some physiological explanations are likely. Neuromuscular blocking agents are rarely useful to treat PPHN since the introduction of iNO, nevertheless, it might still be a useful rescue therapy in some selected newborns with PPHN refractory to classical treatments.
\end{abstract}

Keywords: Premature neonates; Neuromuscular blockade; Refractory persistent pulmonary hypertension

\section{Introduction}

Persistent pulmonary hypertension of the newborn (PPHN) is an abnormal early adaptation to the perinatal circulation transition. Failure of the normal postnatal decline in pulmonary vascular resistance (PVR) leads to right to left intra- or extra-cardiac shunts, which impairs systemic oxygenation and right ventricular failure. PPHN affect 2 to 6 per 1,000 live birth [1]. Mortality rate is still quite high (up to $48 \%$ ) as

\footnotetext{
Manuscript accepted for publication February 21, 2013

${ }^{\text {a}}$ Pediatric and Neonatal Intensive Care Unit, Geneva University Hospital, Switzerland

${ }^{\mathrm{b}}$ Corresponding author: Cristina Delco, Rue Willy-Donze 6, 1211

Geneve 14, Switzerland. Email: cristina.delco@hcuge.ch
}

doi: http://dx.doi.org/10.4021/jmc1114w well as adverse neurological sequelae (up to 46\%) [2].

In the pre inhaled nitric oxide (iNO) era (before 1993) a wide variation in treatment of this entity was noted and one of the possibilities was neuromuscular blockers [3, 4]. We present a patient with PPHN refractory to iNO for whom, neuromuscular blockers were beneficial.

\section{Case Report}

We report the case of a boy baby, born by emergency caesarean section at 27 weeks of gestation because of placental abruption. The patient was born in a regional hospital, but was nonetheless taken care of by trained neonatologist. No antenatal corticosteroids had been administered, membranes were still intact and amniotic fluid was clear. Birth weight was 1,090 g (p 50 - 90), height $36 \mathrm{~cm}$ (p 50 - 90) and head circumference $25.5 \mathrm{~cm}$ (p 50 - 90). Apgar was 5/8/9 and arterial and venous umbilical cord $\mathrm{pH}$ values were 7.37 and 7.45, respectively. Respiratory distress and oxygen requirement up to $60 \%$ were apparent after a few minutes and the baby was intubated at 5 minutes of life. A venous umbilical line was inserted, $10 \mathrm{~mL} / \mathrm{kg}$ of crystalloids was administered because of hypotension (mean arterial pressure $24 \mathrm{mmHg}$ ) and a dose

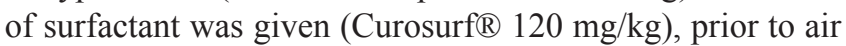
transfer to our tertiary neonatal intensive care unit (NICU). Upon arrival in our unit, seven hours after birth, we put the baby on high frequency oscillation ventilation (HFO), as per our protocol.

At 48 hours of life he developed clinical evidence of severe pulmonary hypertension, with pre- and post-ductal differential saturation up to $60 \%$. Echocardiography revealed a large patent ductus arteriosus (PDA) with bidirectional shunt and tricuspid valve regurgitation through a foramen ovale, confirming PPHN (Fig. 1), iNO was started and the newborn was adequately sedated with a continuous infusion of fentanyl. As sepsis was also suspected because of a metabolic acidosis (pH 7.31, pCO2 $3.9 \mathrm{kPa}$, bic $14 \mathrm{mmol} / \mathrm{L}, \mathrm{BE}-11$, lactate $4 \mathrm{mmol} / \mathrm{L}$ ) associated with leukopenia (total leukocyte count $1.9 \mathrm{G} / \mathrm{L}$ ) and CRP $45 \mathrm{mg} / \mathrm{L}$, antibiotics (ampicillin and gentamycin) were started. There was no parenchymal lung disease on chest X-ray. 


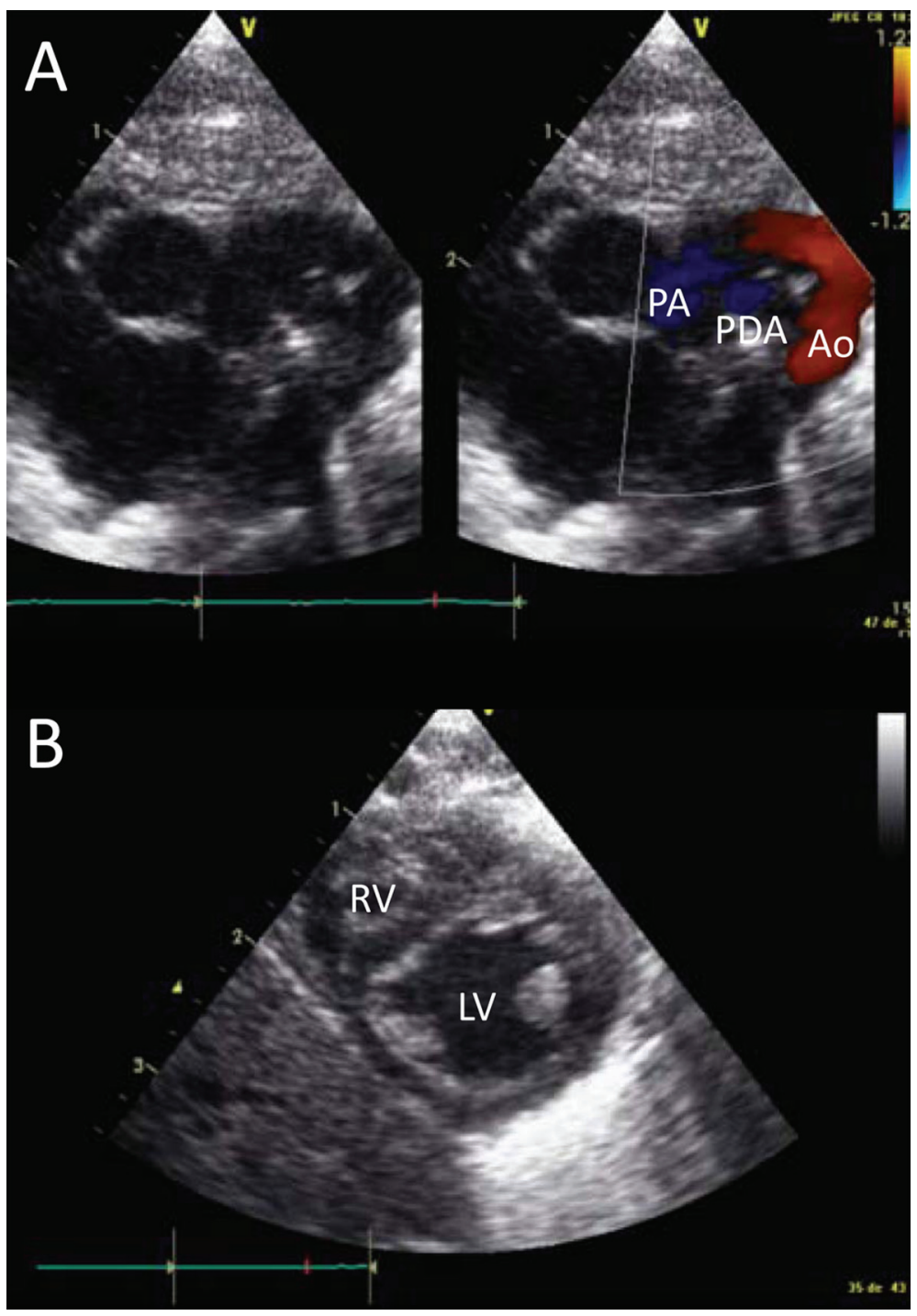

Figure 1. Cardiac ultrasound. Panel A shows the large patent ductus arteriosus (PDA) between the pulmonary artery (PA) and the aorta (Ao). Panel B shows the relationship between the right (RV) and left ventricule (LV), with the interventricular septum protruding in the LV.

Despite intensive care therapy including HFO (mean airway pressure $12 \mathrm{~cm} \mathrm{H}_{2} \mathrm{O}$, amplitude 55 , frequency $15 \mathrm{~Hz}$, after a recruitment manoeuvre), iNO $20 \mathrm{ppm}$, a better control of acid-base balance by reducing $\mathrm{pCO} 2(\mathrm{pH} 7.31, \mathrm{pCO} 24$ $\mathrm{kPa}$ ), $\mathrm{FiO} 250 \%$, sedation and treatment of infection, PPHN worsened (post ductal saturation constantly between 30 and $70 \%$ ) and oxygen requirement did not decrease. At 55 hours of life a bolus of mivacurium was administered $(0.2 \mathrm{mg} / \mathrm{kg}$ intravenously). Within 30 seconds after administration, the post-ductual saturation increased dramatically up to $89 \%$ without any other hemodynamical or respiratory changes. After 10 minutes, the post-ductal saturation decreased again to $62 \%$. To reproduce these encouraging results for a longer period, we gave a single bolus of pancuronium $(0.1 \mathrm{mg} / \mathrm{kg})$. The post-ductal saturation rose again to $90 \%$ (Fig. 2), but the blood pressure and heart rate were unchanged, as was the clinical evaluation (no change in cutaneous perfusion). In the following 48 hours, iNO was weaned and the patient was successfully extubated at Day 5 of life. Due to positive blood cultures for Staphylococcus aureus, we stopped gentamycin after 4 days, and replaced amoxicillin with vancomycin; this treatment was continued for 2 weeks.

\section{Discussion}

We report the case of a premature boy presenting a severe PPHN, induced by an early-onset sepsis, refractory to classical treatments but responsive only to neuromuscular blockade agents. 


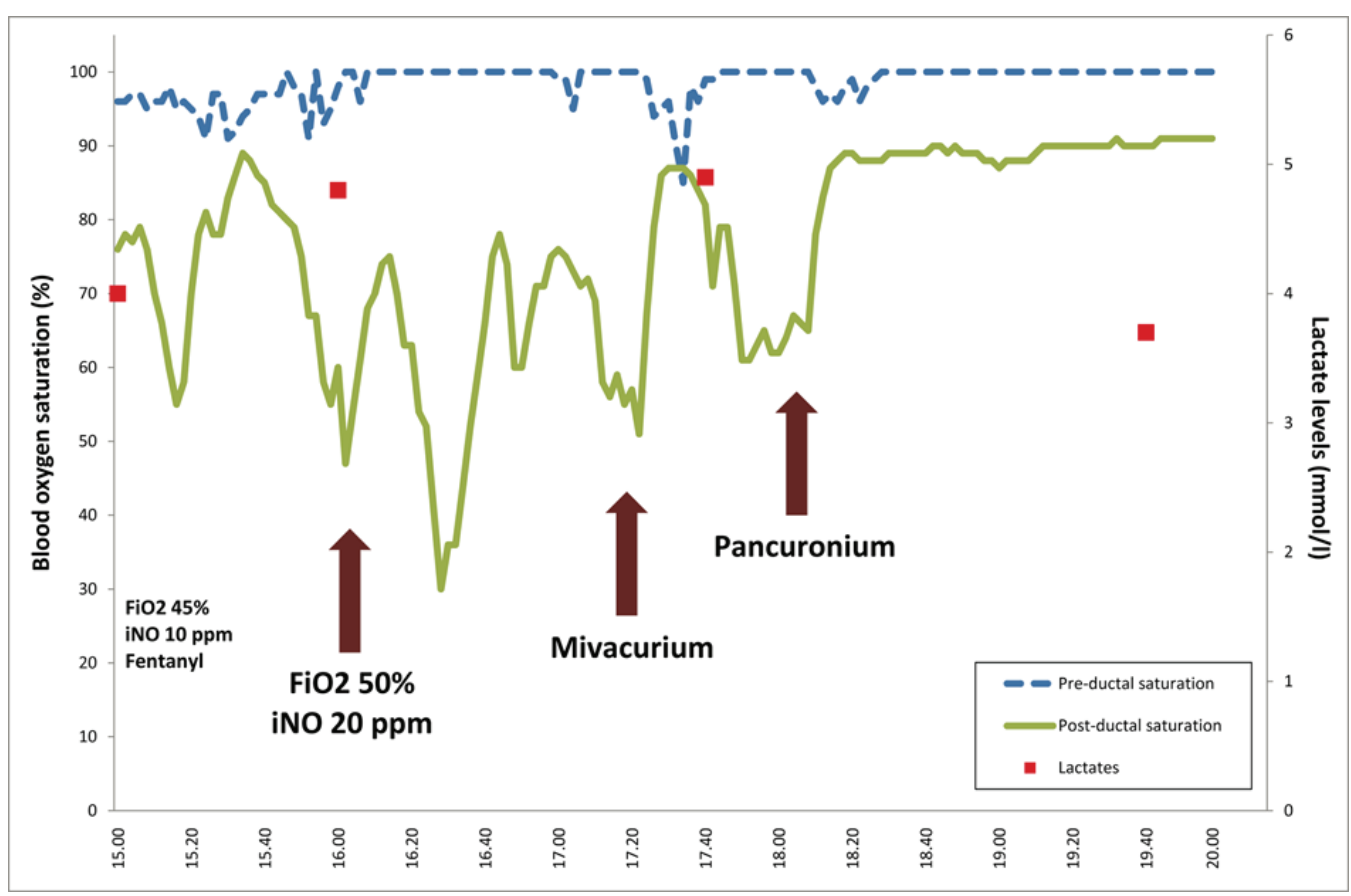

Figure 2. Evolution of the pre- and post-ductal saturation (dashed and full line, respectively) as well as the lactate levels (squares, secondary scale on the right) and the different interventions (vertical arrows), according to the time (horizontal scale).

PPHN is characterized by sustained elevation of PVR; decreased lungs perfusion and continuous right to left intra(through the foramen ovale) or extra- (through the patent ductus arteriosus) cardiac blood shunting, leading to impaired systemic oxygenation and decreased right ventricular function. This may lead to a dilatation of the right ventricule, compressing the left ventricule and decreasing the cardiac output.

PPHN generally presents as one of three physiopathological mechanisms [5]: a structurally normal but abnormally constricted pulmonary vasculature (meconium aspiration syndrome, sepsis, respiratory distress syndrome); a structurally abnormal vasculature following an antenatal remodelling (named "idiopathic PPHN"); or a hypoplastic vasculature (congenital diaphragmatic hernia). As pulmonary vascular tone is mediated by competing vasodilatory and vasoconstrictive factors, the primary aim of therapies is to produce a selective pulmonary vasodilatation.

Despite advances in management of this condition, no single therapeutic approach has been shown to be universally effective in the treatment of PPHN. Therefore practice variations are common [2]. The majority of physicians choose therapies that promote optimal lung inflation with recruitment strategies (namely with HFO and surfactant) and correction of stimuli known to increase PVR (acidosis, polycythaemia, pain).

Second tier treatments specifically target pulmonary vascular tone, such as iNO. Although this therapy is now standard of care in PPHN, 30\% of patients seem to be nonresponders, as in our case [6]. For these patients, rescue therapies with sildenafil, milrinone, prostacyclin or bosen$\tan$ have been reported [7]. However, there is no evidence to validate these rescue therapies.

The use of neuromuscular blockers in the treatment of PPHN with severe hypoxemia has scarcely been described, and solely prior to the "iNO era".

In 1976, Levin et al [8] reported an increasing in $\mathrm{PaO} 2$ after curare administration that they attributed to a more effective ventilation and release of histamine. Henry et al [3] described similar findings and suggested that curare induces respiratory paralysis, which would reduce right to left shunting, hence improving $\mathrm{PaO} 2$ and finally decreasing mortality in infant with severe PPHN. One year later Hutchison and $\mathrm{Yu}$ [4] examined the effect of curare in the treatment of PPHN. Curare decreased PVR in only one infant out of thirteen. However, subsequent analysis revealed that following curare, alveolo-arterial gradient increased statistically, but this was probably not clinically significant.

In our case of refractory PPHN a clear and certain benefit following curare administration was noted, permitting decreasing $\mathrm{FiO} 2$ and ventilator parameters. Some explanations may be raised: firstly this could be related to improved and more effective ventilation due to a better wall compliance and a fully synchronize infant's breathing (decreased struggling) but our patient was comfortable under a fentanyl continuous perfusion and on HFO. The neuromuscular blocker 
could trigger a release of histamine, which in two studies $[9,10]$, has been recognised as a pulmonary vasodilator acting on H2-receptors in pulmonary vasculature in dogs and rats; however our patient did not present any signs of systemic vasodilatation, and pancuronium is known to usually have an overall hypertensive effect. Respiratory paralysis may reduce oxygen consumption by eliminating the work of breathing and may also reduce peak transthoracic pressure decreasing risk of barotrauma, but our patient was ventilated on HFO and was already apneic prior to curare. Finally a thoracic rigidity caused by fentanyl is possible but would not have complicated the ventilation on account of the HFO ventilation. However, by paralysing the diaphragm and the abdominal wall muscles, the intra-abdominal pressure might have decreased, possibly decreasing the intra-thoracic pressure, improving the lung recruitment, decreasing the PVR and hence decreasing the right-to-left shunt. Despite all these hypotheses, a sound physiological mechanism to explain our patient's improvement is still missing.

In conclusion, neuromuscular blocking agents are rarely useful to treat PPHN since the introduction of iNO. Nevertheless, it might still be useful in some selected newborns with PPHN refractory to classical treatments. Further studies are warranted to better understand the implicated mechanisms and to determine the current indications to such a treatment.

\section{Declaration}

There was no sponsorship nor funding involved.

\section{References}

1. Walsh-Sukys MC, Tyson JE, Wright LL, Bauer CR, Korones SB, Stevenson DK, Verter J, et al. Persistent pulmonary hypertension of the newborn in the era before nitric oxide: practice variation and outcomes. Pediatrics. 2000;105(1 Pt 1):14-20.

2. Shivananda S, Ahliwahlia L, Kluckow M, Luc J, Jankov $\mathrm{R}, \mathrm{McNamara}$ P. Variation in the management of persistent pulmonary hypertension of the newborn: a survey of physicians in Canada, Australia, and New Zealand. Am J Perinatol. 2012;29(7):519-526.

3. Henry GW, Stevens DC, Schreiner RL, Grosfeld JL, Ballantine TV. Respiratory paralysis to improve oxygenation and mortality in large newborn infants with respiratory distress. J Pediatr Surg. 1979;14(6):761-767.

4. Hutchson AA, Yu VY. Curare in the treatment of pulmonary hypertension as it occurs in the idiopathic respiratory distress syndrome. Aust Paediatr J. 1980;16(2):94100.

5. Porta NF, Steinhorn RH. Pulmonary vasodilator therapy in the NICU: inhaled nitric oxide, sildenafil, and other pulmonary vasodilating agents. Clin Perinatol. 2012;39(1):149-164.

6. Filippi L, Serafini L, Gozzini E, Poggi C, Moroni M, Fiorini P. Persistent pulmonary hypertension of the newborn refractory to inhaled nitric oxide and prostacyclin, responsive to neuromuscular blockade. Minerva Pediatr. 2011;63(3):233-235.

7. Oishi P, Datar SA, Fineman JR. Advances in the management of pediatric pulmonary hypertension. Respir Care. 2011;56(9):1314-1339; discussion 1339-1340.

8. Levin DL, Heymann MA, Kitterman JA, Gregory GA, Phibbs RH, Rudolph AM. Persistent pulmonary hypertension of the newborn infant. J Pediatr. 1976;89(4):626630 .

9. Tucker A, Weir EK, Reeves JT, Grover RF. Histamine $\mathrm{H} 1$ - and H2-receptors in pulmonary and systemic vasculature of the dog. Am J Physiol. 1975;229(4):1008-1013.

10. Lau WH, Kwan YW, Au AL, Cheung WH. An in vitro study of histamine on the pulmonary artery of the Wistar-Kyoto and spontaneously hypertensive rats. Eur J Pharmacol. 2003;470(1-2):45-55. 